\title{
EXPERIMENTAL INVESTIGATION OF DROPLET EVAPORATION OF SUSPENSION BASED ON WATER WITH SUBSURFACE ADMIXTURES IN A FLAME AREA
}

\author{
Margarita A. Dmitrienko ${ }^{1}$, Olga V. Vysokomornaya, ${ }^{1, a}$, Jean Claude Legros ${ }^{1,2}$, Anastasia A. \\ Shcherbinina ${ }^{1}$ \\ ${ }^{1}$ National Research Tomsk Polytechnic University, 634050 Tomsk, Russia \\ ${ }^{2}$ Université Libre de Bruxelles, 1050 Brussels, Belgium
}

\begin{abstract}
The influence of typical mixtures (clay, sand, soil, silt) on integral characteristics of droplet evaporation of suspension based on water in high-temperature (about $1100 \mathrm{~K}$ ) gases was investigated experimentally by the optical methods and high-speed (to $10^{5}$ frames per second) video recording system. The significant intensification of heat up and evaporation of suspension droplets was defined when varying the relative mass fraction of admixtures within the range $\gamma=0-0.1$ for a sand, $\gamma=0-0.01$ for clay, ground, and silt.
\end{abstract}

\section{Introduction}

Water is one of the most widely accepted process liquids in production cycles of the majority of industries [1,2]. The technical process with applying the water, as well as emulsions and suspensions on its base means very often the heating and evaporation of droplet flows in high-temperature gas mediums in a production cycle: at producing the two-phase gas-vapor heat carriers, in a technology of polydisperse fire fighting, for implementing the thermal treatment of polluted industrial and domestic water and other.

The results of experimental investigations [3] illustrated that the intensity of phase transformation in indicated technological cycles depends largely on characteristic sizes of atomized water droplets and iquid compositions on its base. However, resulting from experimentation [4, 5], it was defined that droplet deceleration, turning and removal by reversed flow of heating gas medium is possible at droplet breaking to characteristic sizes $R_{\mathrm{d}}<200 \mu \mathrm{m}$.

The work [6] shows that the presence of solid admixtures in water droplets, which characterize by big (relative to water) values of heat conduction and temperature conductivity, intensifies the heat up and phase transformations of inhomogeneous droplets. Under specified conditions, the change in thermal and physical characteristics of droplets due to the presence in them of particles of typical soil admixtures, like clay, sand, silt, soil, can be considered as an alternative way for increasing the evaporation completeness.

\footnotetext{
${ }^{\mathrm{a}}$ Corresponding author: vysokomornaja@tpu.ru
} 
The aim of the present work is the determination of integral evaporation characteristics of suspension droplet based on water with typical soil admixtures in a high-temperature combustion product medium.

\section{Experimental setup and procedure}

The setup presented in Figure 1 was used in experimentations.

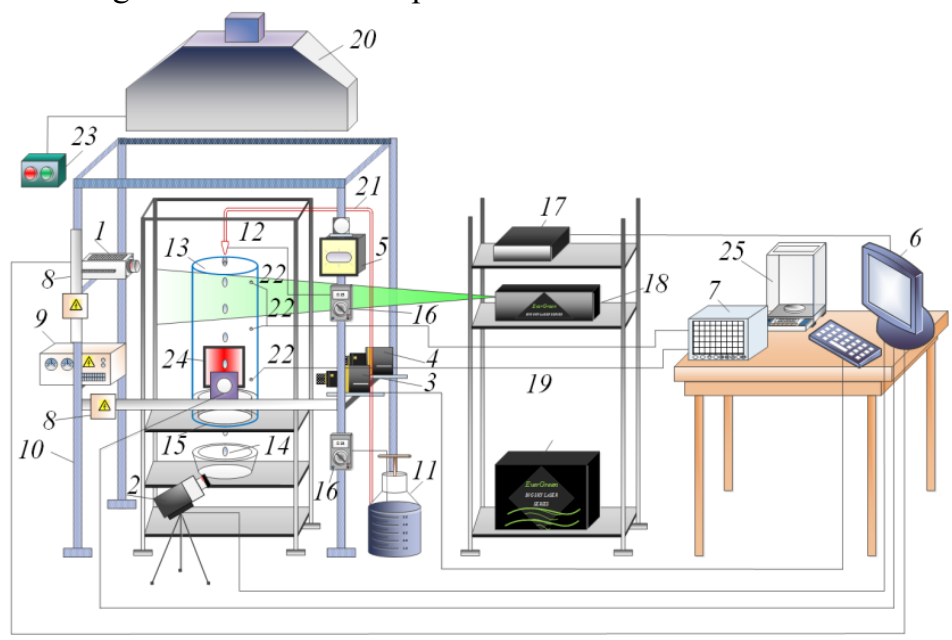

Figure 1. Scheme of experimental setup: 1, 2 - high-speed video cameras; 3, 4 - cross-correlation cameras; 5 spotlight; 6 - personal computer (PC); 7 - technological multichannel registrar; 8 -motorize coordinate device; 9 - power module of motorize coordinate device; 10 - aluminium rack; 11 - vessel with water; 12 - droplet dosing device (atomizer); 13 - quartz glass cylinder; 14 - droplet catcher; 15 - hollow cylinder with combustible liquid; 16 - digital multimeter; 17 - synchronizer of PC, cross-correlation camera and laser; 18 - two-pulse Nd:YAG laser; 19 - generator of laser emission; 20 - plenum system; 21 - supplying channel for water; 22 thermocouples; 23 - on/off remote control of plenum system; 24 - diffusion screen complete with lightguide; 25 - analytic balance system.

Intracavity of cylinder 13 was filled with liquid combustion substance - acetone, which was fired then. At the end of the period necessary for a stable combustion of acetone and channel 13 heat up (about $200 \mathrm{~s}$ ), size-defined droplets of suspension were supplied into high-temperature (about $1100 \mathrm{~K}$ ) combustion products by the dosing device 12 . The trajectory of droplet movement coincided with the symmetry axis of the channel 13 . The process of droplet free falling in high-temperature gases was recorded by high-speed video cameras 1 and 2, and cross-correlation cameras 3 and 4 . To determine the form and the sizes of droplets, the «Tema Automotive» software with the function of continuous keeping track of dynamic object was applied. Position, form and characteristic size of droplets $R_{\mathrm{d}}$, and their motion speeds $U_{\mathrm{d}}$ in a high-temperature combustion product flow was determined. Droplet sizes of video frames (before and after flame zone) in computational domains were defined by means of the video recording presenting a consecutive list of videogrames in a fixed time period.

As the main characteristic of studied process, we used the parameter characterizing the size reduction of droplet at motion through high-temperature gases: $\Delta R=\left(R_{\mathrm{d}}-R_{\mathrm{d}}{ }^{*}\right) / R_{\mathrm{d}}$, where $R_{\mathrm{d}}, R_{\mathrm{d}} *$ is a value of the conditional mean radius of the droplet at the inlet and outlet from high-temperature gas medium, mm. Systematic errors of droplet radius measurement by high-speed cameras 1 and 2 and corresponding software at $S=0.025-0.04 \mathrm{~mm} /$ pixel did not exceed $0.03 \mathrm{~mm}$.

\section{Results and discussion}

Figure 2 illustrates dependences of parameter $\Delta R$ change in relative mass fraction of admixtures $\gamma$. 


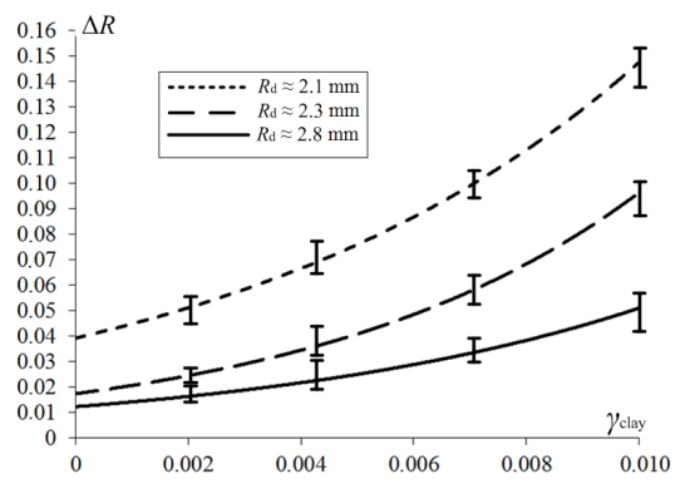

(a)

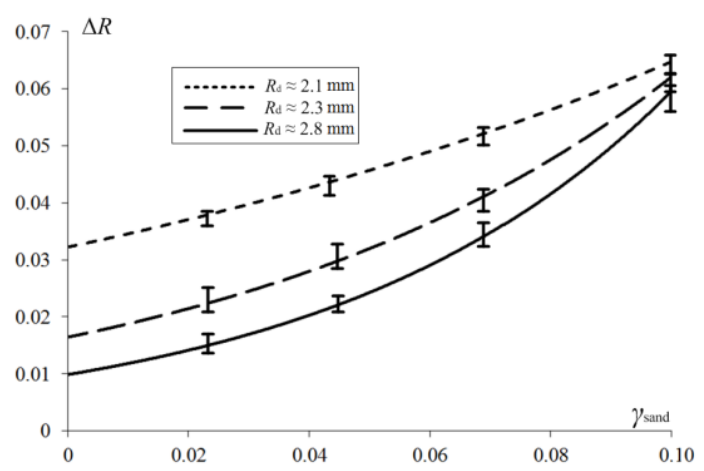

(c)

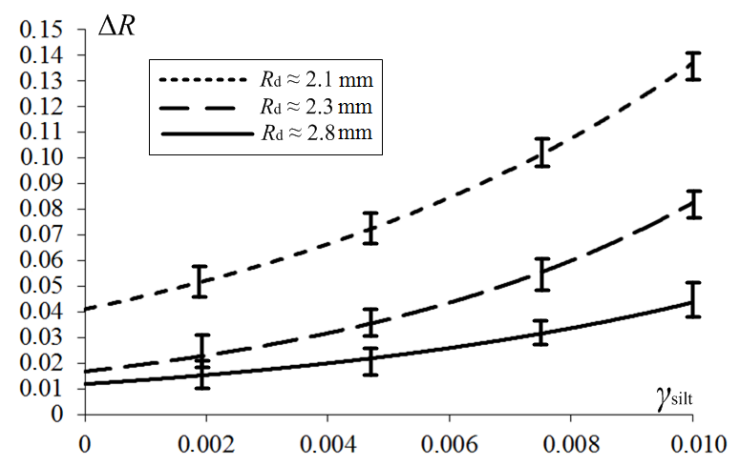

(b)

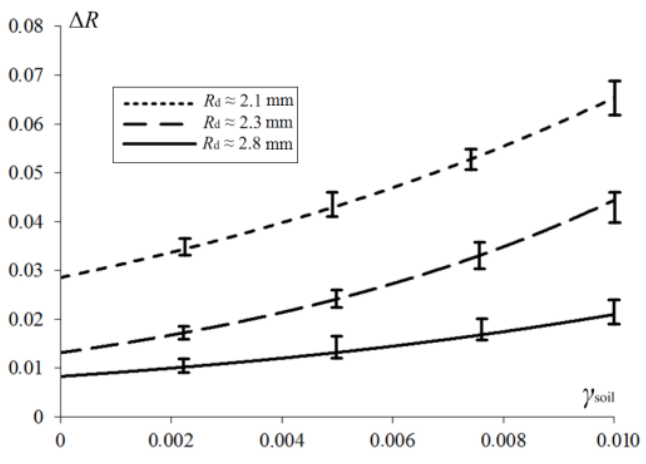

(d)

Figure 2. Dependences of $\Delta R$ on concentration of clay $\left(\gamma_{\text {clay }}\right)(\mathrm{a})$, silt $\left(\gamma_{\text {silt }}\right)(\mathrm{b})$, sand $\left(\gamma_{\text {sand }}\right)(\mathrm{c})$, soil $\left(\gamma_{\text {soil }}\right)(\mathrm{d})$ in water for single droplets of suspension with different initial mean radii $R_{\mathrm{d}} \mathrm{mm}$ at motion $\mathrm{n}$ through hightemperature combustion products.

Analysis of obtained dependences allowed concluding that their presence intensifies significantly the process of suspension droplet evaporation. This effect manifested particularly large-scale in experiments by using silt, clay and soil for suspension preparation. This is due to that fact that thermophysical (heat conduction $\lambda$, heat capacity $c$ and temperature conductivity $a$ ) and optical characteristics of the suspension are higher than of «homogeneous" or «pure» water (without admixtures). Such kinds of admixtures absorb and store a radiant energy of the flame (combustion products), that serve to an accelerated (relatively to water without admixtures) heat and evaporation of droplets, because it takes less energy to heat the boundary layer of suspension droplet up to conditions of intensive evaporation. The mechanism of this influence is described sufficiently detailed for aluminium and carbon in [7]. In a case of sand and clay, physical mechanism of influence of such substance particles is similar to that presented in [7], but the intensity of absorption processes of heat entering to the «water - sand» interface is much higher than in a case of «carbon - water» mixture. The energy incoming to a surface of single particle due to radiative heat transfer (opposite to carbon or aluminium) is accumulated in a thin water layer bearing against the surface. In addition, it was established [3] that particles of water and other liquids are deformed intensively during the motion through high-temperature gases. Resulting such deformations, an intense mixing of «hot» and relatively«cold» layers of water in a suspension droplet occurs. The mean temperature of suspension droplet increases much faster than under conditions of deformation of homogeneous liquid (for example, water) droplet. If there are some (a group) particles of sand in a droplet, the processes of heat exchange between individual layers of liquid are even more intensified.

Approximating expressions were obtained for dependences presented in Figure 2. The expressions for suspensions included clay (1)-(3) and sand (4)-(6) can be mentioned as typical ones. 
When initial radius is $R_{\mathrm{d}} \approx 2.1 \mathrm{~mm}$ :

$\Delta R=3.916 \mathrm{e}^{1.3264 \gamma \text { clay }}, 0<\gamma_{\text {clay }}<0.01, U_{\mathrm{d}} \approx 3 \mathrm{~m} / \mathrm{s}, T_{\mathrm{w}} \approx 300 \mathrm{~K}$.

When initial radius is $R_{\mathrm{d}} \approx 2.3 \mathrm{~mm}$ :

$\Delta R=1.7333 \mathrm{e}^{1.7158 \gamma_{\text {clay }}}, 0<\gamma_{\text {clay }}<0.01, U_{\mathrm{d}} \approx 3 \mathrm{~m} / \mathrm{s}, T_{\mathrm{w}} \approx 300 \mathrm{~K}$.

When initial radius is $R_{\mathrm{d}} \approx 2.8 \mathrm{~mm}$ :

$\Delta R=1.2241 \mathrm{e}^{1.4271 \gamma \text { clay }}, 0<\gamma_{\text {clay }}<0.01, U_{\mathrm{d}} \approx 3 \mathrm{~m} / \mathrm{s}, T_{\mathrm{w}} \approx 300 \mathrm{~K}$.

When initial radius is $R_{\mathrm{d}} \approx 2.1 \mathrm{~mm}$ :

$\Delta R=3.2229 \mathrm{e}^{0.0698 \gamma \text { sand }}, 0<\gamma_{\text {sand }}<0.01, U_{\mathrm{d}} \approx 3 \mathrm{~m} / \mathrm{s}, T_{\mathrm{w}} \approx 300 \mathrm{~K}$.

When initial radius is $R_{\mathrm{d}} \approx 2.3 \mathrm{~mm}:$

$\Delta R=1.6434 \mathrm{e}^{0.133 \gamma \mathrm{sand}}, 0<\gamma_{\text {sand }}<0.01, U_{\mathrm{d}} \approx 3 \mathrm{~m} / \mathrm{s}, T_{\mathrm{w}} \approx 300 \mathrm{~K}$.

When initial radius is $R_{\mathrm{d}} \approx 2.8 \mathrm{~mm}$ :

$\Delta R=0.9868 \mathrm{e}^{0.1799 \gamma \mathrm{sand}}, 0<\gamma_{\text {sand }}<0.01, U_{\mathrm{d}} \approx 3 \mathrm{~m} / \mathrm{s}, T_{\mathrm{w}} \approx 300 \mathrm{~K}$.

Presented approximations (1)-(6) allow calculating the change of characteristic size of the droplet at varying the concentration of clay and sand in ranges typical for technologies of water thermal treatment.

\section{Conclusion}

As a result of conducted experimental investigations, the laws of evaporation intensification of water containing admixtures of sand, clay, soil, silt in high-temperature gas mediums were analyzed. Obtained experimental dependences illustrates significantly more intense heat up and droplet evaporation of suspension compared to water without admixtures at varying their relative mass fraction within a short range $(\gamma=0-0.1$ for sand, $\gamma=0-0.01$ for clay, soil and silt). Defined effect is due to a difference of heat up and energy accumulation of gases by suspensions in comparison with homogeneous water. It shows that the efficiency of high-temperature water treatment is higher, the stronger concentration of admixtures is.

This work was supported by the Russian Foundation for Basic Research (14-08-00057). The change of characteristic size of droplet suspensions was performed with financial support of the scholarship of the President of the Russian Federation for young scientists (SP-1350.2015.1).

\section{References}

1. M.I. Shilyaev, E.M. Khromova, Theor. Found. Chem. Eng. 4, 42 (2008).

2. V.N. Babak, Theor. Found. Chem. Eng. 3, 39 (2005).

3. R.S. Volkov, G.V. Kuznetsov, P.A. Strizhak, Theor. Found. Chem. Eng. 4, 49 (2015).

4. O.V. Vysokomornaya, G.V. Kuznetsov, P.A. Strizhak, Fire Saf. Journ. 70 (2014).

5. R.S. Volkov, G.V. Kuznetsov, P.A. Strizhak, Int. J. of Heat and Mass Transf. 79 (2014).

6. I.S. Anufriev, G.V. Kuznetsov, M.V. Piskunov, P.A. Strizhak, M.Yu. Chernetskii. Tech. Phys. Lett. 8, 41 (2015).

7. R.S. Volkov, G.V. Kuznetsov, P.A. Strizhak, Int. J. of Therm. Science. 88 (2015). 